\title{
Review of: "Evaluation of multi-task learning in deep learning-based positioning classification of mandibular third molars"
}

\author{
József Szalma ${ }^{1}$
}

1 University of Pecs

Potential competing interests: The author(s) declared that no potential competing interests exist.

First, I would like to congratulate on the work of Sukegawa et al., comparing two CNN deep learning models for determining Pell-Gregory $(P \& G)$ and Winter classification of impacted third molars.

Although, these classifications were constructed almost hundred years before, oral surgeons still use them in the everyday practice. One can hardly find published research about impacted third molars without describing and characterizing the examined study populations with these classifications. Furthermore, many investigations found correlations with some impaction depth or angulation type and surgical removal's difficulty, mandibular fracture pattern, inferior alveolar nerve injury or development of local pathologic lesions.

I think, the significance of this work is mostly in the potential that later, artificial intelligence determined classification and scoring values can be automatically combined with existing literature data. I mean, some dedicated software automatically calculates $P \& G$ and Winter's values, and immediately give some report about odds ratios of already published and known consequences. E.g., the seen impaction pattern and its correlation with angle/condyle fractures with some concrete odds [1]. Or the kind of impaction pattern which was estimated and its correlation with second molar distal surface complications (periodontal inflammation or bone loss or caries etc.) [2]. Or to mention immediately the significantly longer postoperative discomfort which was found with the current type of impaction [3].

This would mean that dentists without significant experience in third molar surgery, or patients after panoramic radiography would have significantly more, faster, and personalized information regarding the actual case on an automated basis.

Although, P\&G and Winter's classifications are important part of risk estimation prior third molar surgery, they estimate the situation only in 2 dimensions and only in the sagittal plane. Several other general (age, bone metabolism, medications, immunsuppression etc.) and local factors (mouth opening, number and shape of the roots, relation to the inferior alveolar canal and, space between third and second molars etc.) should also be considered for a correct analysis of course.

Lastly, I would like to represent two cases, about one of the limitations regarding abovementioned classifications. Both illustrated cases had a very similar impaction status (P\&G II/A) and angulation (distoangular), however, e.g., the risk for inferior alveolar nerve injury was significantly different. In the 
case left, marked distance between third molar's roots and inferior alveolar canal (IAC) exist (yellow-black arrow), however, in the case on the right roots were superimposed on the IAC, showing one of the so-called specific "classic" "high-risk" signs, 'interruption of the superior border of the IAC' (arrow indicating the lower cortex of the IAC).

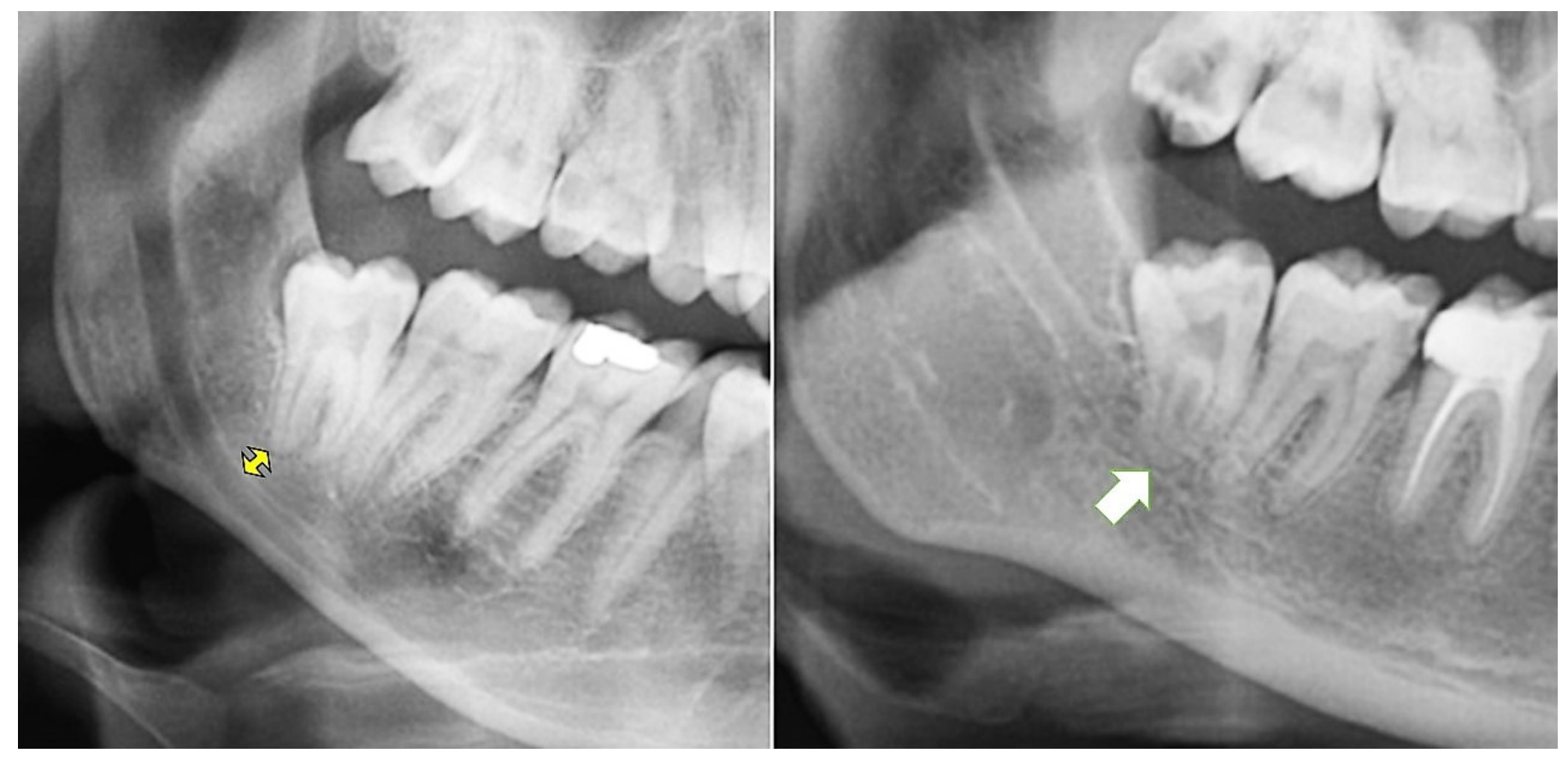

References

[1.] Soós B, Janovics K, Tóth Á, Di Nardo MD, Szalma J. Association Between Third Molar Impaction Status and Angle or Condylar Fractures of the Mandible: A Retrospective Analysis. J Oral Maxillofac Surg. 2020; 78: 1162.e1-1162.e8. doi: 10.1016/j.joms.2020.02.005.

[2.] Butzin S. To prophylactically extract or not to extract partially erupted mesio-angularly impacted lower third molars? Br Dent J. 2021; 231: 445-448. doi: 10.1038/s41415-021-3561-3

[3.] Vranckx M, Lauwens L, Moreno Rabie C, Politis C, Jacobs R. Radiological risk indicators for persistent postoperative morbidity after third molar removal. Clin Oral Investig. 2021; 25: 4471-4480. doi: 10.1007/s00784-020-03759-7. 